\title{
Diquat and Paraquat Analysis in Rice using a Zwitterionic Hydrophilic Interaction Chromatography - tandem Mass Spectrometry
}

\author{
Nguyen Thanh-Nho a, ${ }^{\mathrm{*}}$, Nguyen Nhu-Quynh ${ }^{\mathrm{b}}$, Nguyen Cong Hau ${ }^{\mathrm{a}}$ \\ ${ }^{a}$ Faculty of Environmental and Food Engineering, Nguyen Tat Thanh University, Ho Chi Minh City, Vietnam \\ ${ }^{\mathrm{b}}$ Center of Analytical Services Experimentation of Ho Chi Minh City, Vietnam \\ * corresponding author: ntnho@ntt.edu.vn \\ DOI : 10.20885/ijca.vol5.iss1 .art4
}

\begin{tabular}{|c|c|}
\hline ARTICLE INFO & ABSTRACT \\
\hline $\begin{array}{l}\text { Received : } 27 \text { November } 2021 \\
\text { Revised : } 26 \text { December } 2021 \\
\text { Published : 01 March } 2022 \\
\text { Keywords : diquat, paraquat, rice, } \\
\text { HILIC, UPLC-MS/MS }\end{array}$ & $\begin{array}{l}\text { The analysis of polar herbicides by liquid chromatography-tandem } \\
\text { mass spectrometry (LC-MS/MS) is challenging due to their } \\
\text { complicated interactions. Diquat and paraquat, the bipyridilium } \\
\text { group of polar herbicides, are widely used worldwide. This study } \\
\text { aimed to optimize analytical conditions of diquat and paraquat in } \\
\text { rice matrix using a hydrophilic interaction liquid chromatography } \\
\text { column (Obelisc R; } 2.1 \times 150 \mathrm{~mm}, 5 \mu \mathrm{m}) \text {. The mixtures of } \\
\text { acetonitrile and ammonium formate buffer in water were used as } \\
\text { mobile phase. All buffer solutions were acidified to a pH of } 2.5 \\
\text { using concentrated formic acid. The results showed that } 10 \mathrm{mM} \\
\text { buffer concentration of ammonium formate was optimal compared } \\
\text { to others, including } 5,20 \text {, and } 50 \mathrm{mM} \text {. A mixture of methanol and } \\
\text { water (6:4, v/v) containing } 1 \% \text { formic acid was used as an } \\
\text { extractable solvent for sample preparation. Method quantitation } \\
\text { limit (MQL) of diquat and paraquat in rice samples was } 10 \mu \mathrm{g} / \mathrm{kg} \text {. } \\
\text { Recoveries at different concentrations (i.e., } 20,50 \text {, and } 100 \mu \mathrm{g} / \mathrm{kg} \text { ) } \\
\text { were higher than } 70 \% \text { for diquat, and } 80 \% \text { for paraquat with RSD } \\
<20 \% \text {, respectively. Diquat and paraquat in rice samples } \\
\text { purchased from the local market were lower than MQL, with good } \\
\text { recoveries for standard spiked samples. }\end{array}$ \\
\hline
\end{tabular}

\section{INTRODUCTION}

Diquat (1.1'-ethylene-2,2'- bipyridinium ion) and paraquat (1,1'-dimethyl-4,4'-bipyridinium ion) are fast-acting and non-selective contact quaternary ammonium herbicides $[1,2]$. They are highly polar compounds, which have permanent ionic character and high hydrophilicity. Log $\mathrm{K}_{\mathrm{ow}}$ of diquat and paraquat are -4.5 and -3.05 , respectively [3]. Due to their polar properties, diquat and paraquat are not retained on the reverse-phase chromatographic column. Currently, there are two ways to determine diquat and paraquat using Liquid Chromatography-tandem Mass Spectrometry (LC-MS/MS), including i) using an agent to pair with diquat and paraquat (ion-pairing technique), which is used commonly to analyze ionic compounds [4, 5]. For the ion-pairing technique, diquat and paraquat can pair with many agents. However, when using the liquid chromatography electrospray ionization tandem mass spectrometry (LC-ESI-MS), the reagents must be volatile compounds such as heptafluorobutyric acid (HFBA), pentafluoropropionic acid (PFPA), or trifluoroacetic acid (TFA) [6]. There are two major disadvantages to the ion-pairing technique. 
Firstly, the LC-MS/MS system can be contaminated by the pairing reagents that change the selectivity on the stationary phase surface of the column, causing "baseline increasing" [7]. Secondly, it causes signal 'suppression' in MS, resulting in low sensitivity [7, 8]; ii) direct analysis of diquat and paraquat without ion-pairing by using hydrophilic interaction liquid chromatography (HILIC) column $[7,9]$. In recent years, scientists have been interested in HILIC due to its widely applications in pharmaceuticals, biology, medicine, environment, food, etc. [10]. It is increasingly being used for the analysis of highly polar, hydrophilic compounds [11]. There are many types of HILIC columns with different stationary phase structures developed. Due to two positive charges in the structural formula of diquat and paraquat, the surface of the stationary phase is negatively charged and/or containing a zwitterion has special features that will retain these compounds, i.e., simultaneous analysis of cationic, anionic, and neutral compounds. One of the zwitterionic HILIC columns commonly used to analyze diquat and paraquat is ZIC-HILIC [7] or Obelisc R [12]. Multimechanical retention extends the applications of those columns. However, that causes many difficulties in method development because of its complexity. Therefore, direct analysis overcomes the disadvantages of the previous ion-pairing technique should be paid more attention. In different cultivation stages, to improve rice production yield, herbicides are used increasingly day by day, especially diquat and paraquat. They must be controlled and monitored because of their negative effects on human health and environments. According to European Union, the maximum residue limits (MRLs) for diquat and paraquat requested in rice were at 0.02 and $0.05 \mathrm{mg} / \mathrm{kg}$, respectively [13]. Due to the increasingly strict regulations of rice importing countries as well as ensuring the safety of consumer health, the analytical issue for the trace content of paraquat and diquat in rice needs more attention by the analyst and manager.

The present study aimed to optimize essential parameters for diquat and paraquat analysis without ion-pairing using hydrophilic interaction liquid chromatography column (Obelisc R; $2.1 \mathrm{x}$ $150 \mathrm{~mm}, 5 \mu \mathrm{m}$ ) tandem mass spectrometry on Thermo Scientific TSQ Quantiva LC-MS. Then, the method was applied to analyze diquat and paraquat in rice samples (the staple food for more than haft of the world's population) [14]. Rice samples were chosen because their consumption in recent decades has increased dramatically [15]. The sample preparation method in this study was based on the method of quick polar pesticides (QuPPe) of the European Union Reference Laboratory for Pesticides Requiring Single Residue Methods [14] to analyze rice samples from local markets.

\section{METHOD}

\subsection{Chemicals and equipment}

A stock solution of $1000 \mathrm{mg} / \mathrm{L}(99 \%)$ of diquat dibromide and paraquat dichloride in water, packed $1 \mathrm{~mL}$ in ampules, was purchased from Restek. Acetonitrile (LC-MS grade, 99.9\%), methanol (LC-MS grade, 99.9\%), and formic acid (analytical grade, 99\%) were purchased from Merck. Ammonium formate (98\%) was acquired from Fluka. Primary secondary amine (PSA) $-40 \mu \mathrm{m}$ and octadecyl (C18) - $40 \mu \mathrm{m}$ were supplied from Agilent. A system of UPLC-MS/MS was used, including a Vanquish ${ }^{\mathrm{TM}}$ UPLC (ultra-performance liquid chromatography) coupled to a TSQQuantiva triple quadrupole mass spectrometer (Thermo, USA).

\subsection{Preparation of chemicals}

\section{Standard solutions}

A standard solution of $100 \mathrm{mg} / \mathrm{L}$ of diquat and paraquat, stability up to 24 months, was prepared by diluting $1 \mathrm{~mL}$ of stock solution $1000 \mathrm{mg} / \mathrm{L}$ into $10 \mathrm{~mL}$ using deionized water. A standard solution of $1 \mathrm{mg} / \mathrm{L}$ of diquat and paraquat was obtained by diluting the standard solution $100 \mathrm{mg} / \mathrm{L}$ in methanol containing $1 \%$ formic acid that was stable for at least one month.

The working standard solutions at various concentrations of $1,5,10,25,50,100$, and $200 \mu \mathrm{g} / \mathrm{L}$ were prepared by diluting standard solution $1 \mathrm{mg} / \mathrm{L}$ in a methanol-water mixture $(6: 4, v / v)$ containing $1 \%$ formic acid, respectively. These solutions were prepared freshly before the analysis. We noted that all standard solutions were stored in polypropylene bottles or plastic vials and put in the fridge at $4^{\circ} \mathrm{C}$. All solutions were taken out from the refrigerator and reached room temperature before use. 


\section{Mobile phases}

The LC mobile phase involving two elute solvents were $i$ ) buffer solutions of formic acid/ammonium formate at different concentrations in water (solvent $\mathbf{A}$ ) and ii) acetonitrile (LC-MS grade, 99.9\%) (solvent B). For solvent A, different buffer concentrations of formic acid/ammonium formate solution were investigated at levels of $5,10,20$, and $50 \mathrm{mM}$. To prepare those solutions, we weighed $0.315 \mathrm{~g}, 0.63 \mathrm{~g}, 1.26 \mathrm{~g}$, and $3.15 \mathrm{~g}$ of ammonium formate corresponding to the buffer concentration of $5,10,20$, and $50 \mathrm{mM}$, into the beakers of $1000 \mathrm{~mL}$, respectively. The deionized water was added up to two-third of the beaker volume and stirred. Concentrated formic acid was slowly added to reach desired $\mathrm{pH}$ (i.e., $\mathrm{pH}$ values of buffer solutions were selected for investigation, including 2.5,3.0, and 5.0). Then, the solutions were transferred into a volumetric flask and adjusted to the mark of $1000 \mathrm{~mL}$ using deionized water.

\subsection{Optimization of UPLC-MS/MS}

A standard solution of $1 \mathrm{mg} / \mathrm{L}$ in $\mathrm{MeOH}$ was directly injected into the system. The injection volume was $5 \mu \mathrm{L}$. The column temperature was set at $40{ }^{\circ} \mathrm{C}$. The flow rate of gradient elution was performed at $0.4 \mathrm{~mL} / \mathrm{min}$. Mass spectrometry optimized parameters were presented in Table 1 . The chromatographic separation of diquat and paraquat was accomplished on an Obelisc R column (150 $\times 2.1 \mathrm{~mm}, 5 \mu \mathrm{m})$ with the stationary phase and interaction described in Figure 1, provided from SIELC Technologies (USA).

Standard solutions of $10 \mu \mathrm{g} / \mathrm{L}$ were injected in triplicate on UPLC-MS/MS to select the optimized buffer concentration and $\mathrm{pH}$ of solvent $\mathrm{A}$, respectively. The injection volume was $5 \mu \mathrm{L}$ for each one. We noted that the $\mathrm{pH}$ values were investigated at 2.5, 3.0, and 5.0 based only on previously optimized buffer concentrations. The gradient elutions are exhibited in Figure 2.

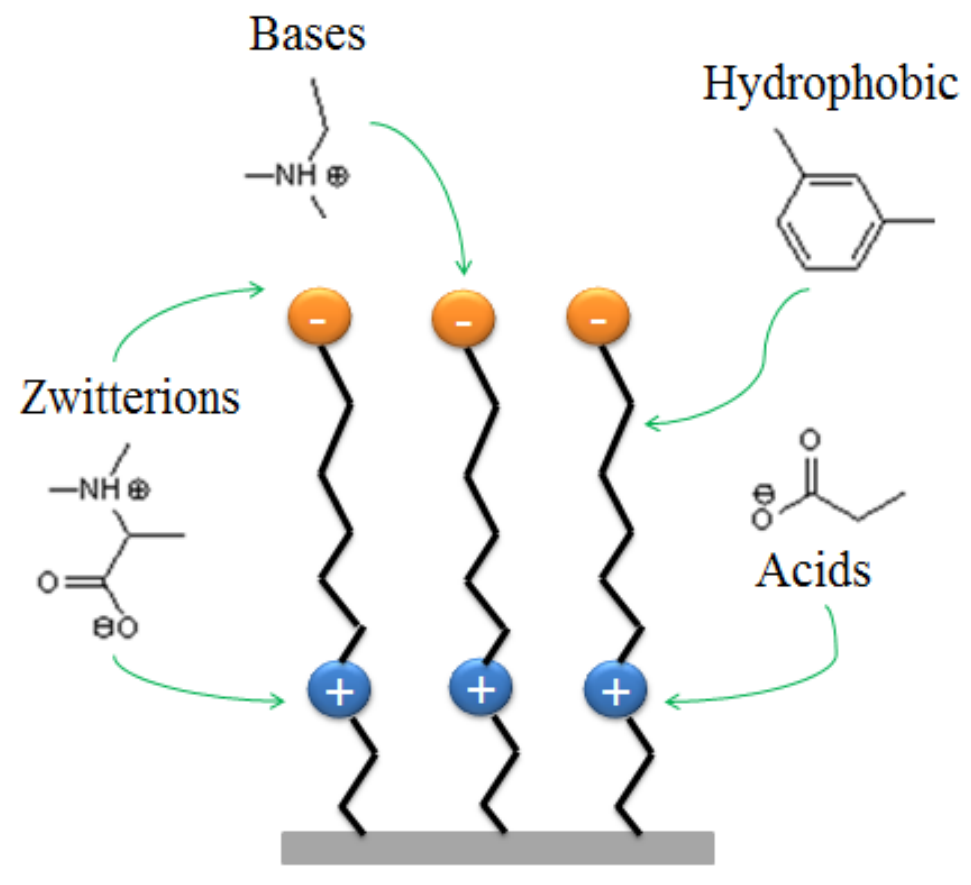

Figure 1. The stationary phase structure and interaction mechanism on Obelisc R column adapted from SIELC [16]. 
TABLE I. Optimized parameters of mass spectrometry

\begin{tabular}{ll|ll}
\hline Ion Source & & Master Scan & \\
\hline Ion Source Type & H-ESI & Use Cycle Time & TRUE \\
Spray Voltage & Static & Cycle Time (s) & 1 \\
Positive Ion (V) & 3500 & Q1 Resolution (FWHM) & 0.7 \\
Negative Ion (V) & 2500 & Q3 Resolution (FWHM) & 0.7 \\
Sheath Gas (Arb) & 45 & CID Gas (mTorr) & 2 \\
Aux Gas (Arb) & 13 & Chrom Filter (sec) & 3 \\
Sweep Gas (Arb) & 1 & Display Retention Time & TRUE \\
Ion Transfer Tube Temp $\left({ }^{\circ} \mathrm{C}\right)$ & 350 & Use Calibrated RF Lens & FALSE \\
Vaporizer Temp $\left({ }^{\circ} \mathrm{C}\right)$ & 350 & Display Retention Time & TRUE \\
\hline
\end{tabular}

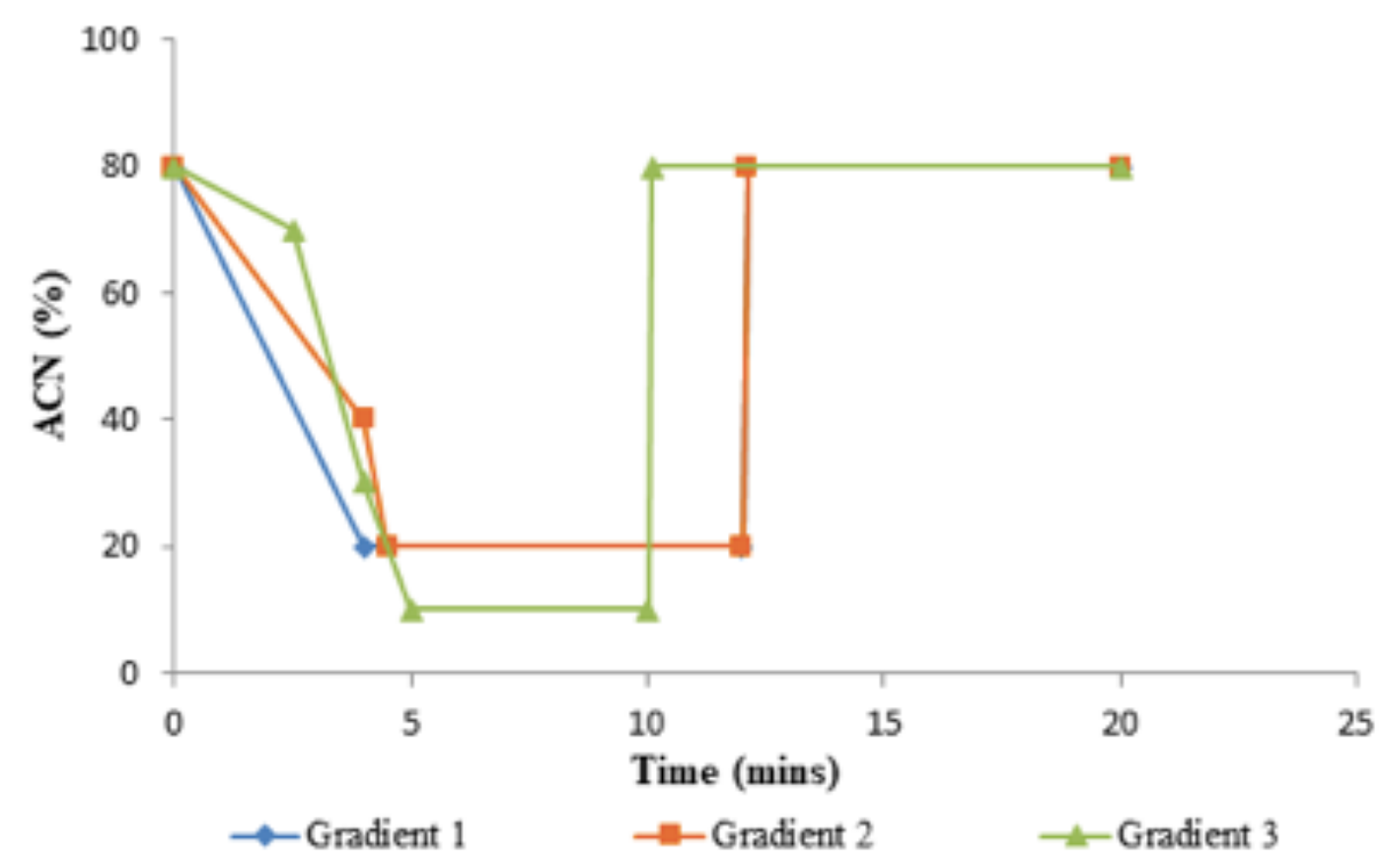

Figure 2. The diagram of gradient elution: buffer solutions of formic acid/ammonium formate at different concentrations in water (solvent A) and ACN-acetonitrile (solvent B).

\subsection{Method validation}

Calibration curves, linearity, and matrix effects

The linearity of analytical curves and matrix effects were determined through injections of the standard solutions at concentrations of $1,5,10,25,50,100$, and $200 \mu \mathrm{g} / \mathrm{L}$ in the mixture of methanol: water $(6: 4=v / v)$ and rice sample with free diquat and paraquat, respectively.

\section{Estimation of method quantitation limit (MQL)}

According to SANTE/11813/2017, MQL is the lowest standard spiked concentration which the analytical method can quantify at levels of repeatability $\left(\mathrm{RSD}_{\mathrm{r}}\right)$, reproducibility $\left(\mathrm{RSD}_{\mathrm{R}}\right)$ less than $20 \%$, recovery between 70 and $120 \%$. For this standard, estimation of method detection limit (MDL) is not mentioned. Thus, in the present study, the formula MDL=3/10 MQL was applied to calculate MDL. Twenty rice samples were spiked at the concentration of $10 \mu \mathrm{g} / \mathrm{kg}$ to determine the MQL of diquat and paraquat. We noted that the samples were investigated on two different days, carrying out 10 samples per day. The recoveries of diquat and paraquat were calculated based on a calibration curve in the solvent. Results of 10 samples on the same day and 20 samples in two days were used to determine $\mathrm{RSD}_{\mathrm{r}}$ and $\mathrm{RSD}_{\mathrm{R}}$, respectively. 


\section{Recovery, repeatability, and reproducibility}

The repeatability, reproducibility, and recovery efficiency of diquat and paraquat were acquired via standard solutions spiked at three levels of 20,50 , and $100 \mu \mathrm{g} / \mathrm{kg}$ into rice samples with free diquat and paraquat. Each investigated concentration was carried out 12 times of repeatability per rice sample on two different days (i.e., 6 samples per day). The recovery of 6 samples on the same day and the 12 samples on two different days were used to determine $\mathrm{RSD}_{\mathrm{r}}$ and $\mathrm{RSD}_{\mathrm{R}}$, respectively.

\subsection{Sample preparation for diquat and paraquat in rice samples}

Five rice samples were gathered from local markets (Ho Chi Minh City, Viet Nam) and were homogenized using a mill. The rice sample preparations were carried out based on the QuPPe method that the European Union Reference Laboratory developed for Pesticides Requiring Single Residue Methods [17]. Briefly, $2 \mathrm{~g}$ of rice was weighed in triplicates into $50 \mathrm{~mL}$ plastic centrifuge tubes with screw caps, $10 \mathrm{~mL}$ mixture of methanol: water $(6: 4=v / v)$ containing $1 \%$ formic acid was added to each tube. These samples were vortexed for 10 seconds and sonicated for 15 minutes at room temperature. The samples were centrifuged for 3 minutes at $9000 \mathrm{rpm}$. Then, $6 \mathrm{~mL}$ of raw extracts were transferred into $15 \mathrm{~mL}$ plastic centrifuge tubes containing sorbent material (150 mg PSA) to remove the matrix. The cleaning process was performed by vortexing the tubes for 30 seconds and centrifuging for 3 minutes at $9000 \mathrm{rpm}$. The cleaned extracts were filtered through a $0.22 \mu \mathrm{m}$ nylon filter into a polypropylene autosampler vial prior to analysis. For the not detected sample of diquat and paraquat, a standard solution of $100 \mu \mathrm{g} / \mathrm{kg}$ was spiked in triplicates and performed according to the above process to evaluate the accuracy of the method.

\section{RESULT AND DISCUSSION}

\subsection{Quantitative and qualitative ions of diquat and paraquat}

The triple quadrupole mass spectrometer on the system of UPLC-MS/MS in the present study was acquired in the positive electrospray ionization mode $\left(\mathrm{ESI}^{+}\right)$. The optimized voltages of collision energy (CE) and radio frequency (RF) lens are presented in Table 2. Two transitions per analyte were monitored in the multiple reaction monitoring (MRM) mode for diquat $(183.12 \rightarrow 157.0$ for quantification; $183.12 \rightarrow 167.9$ for confirmation) and paraquat $(186.33 \rightarrow 170.9$ for quantification; $171.12 \rightarrow 155.1$ for confirmation). It can be seen that precursor ions of diquat have the fragmentation mechanism was $\left[\mathrm{M}^{2+}\right] \rightarrow\left[\mathrm{M}^{\bullet+}\right]$. For paraquat, mass 186.33 has the fragmentation mechanism similar to precursor ions of diquat, as for mass 171.12 was formed by the mechanism $\left[\mathrm{M}^{2+}\right] \rightarrow\left[\mathrm{M}^{+}-\mathrm{CH}_{3}\right][18]$ (Figure 3). After the precursor ions pass through the first quadrupole to the second, they were fragmented to form daughter ions. For paraquat, two precursor ions with m/z of 186.33 and 171.12 lose one $-\mathrm{CH}_{4}$ group to create two fragments with $\mathrm{m} / \mathrm{z}$ of 170.9 and 155.1 [3]. Unlike paraquat, the daughter ion of diquat has the $\mathrm{m} / \mathrm{z}$ of 157.0 , which was formed by [Diquat ${ }^{\bullet+}$ ] breaking an $-\mathrm{HCN}$ group in the aromatic ring [3], as for the $\mathrm{m} / \mathrm{z}$ of 167.9 was formed by [Diquat ${ }^{\bullet+}$ ] losing a group of $\mathrm{CH}_{4}$.

TABLE II. Optimized collision energy (CE) and radio frequency (RF) lens voltages for qualitative and quantitative ions of diquat and paraquat

\begin{tabular}{lcccc}
\hline Compounds & Precusor ion $(\mathrm{m} / \mathrm{z})$ & Product ion $(\mathrm{m} / \mathrm{z})$ & CE $(\mathrm{V})$ & RF lens $(\mathrm{V})$ \\
\hline Diquat & 183.12 & $157.0^{*}$ & 22.2 & 79 \\
& 183.12 & 167.9 & 28.6 & 79 \\
Paraquat & & & & \\
& 186.33 & $170.9^{*}$ & 19.6 & 56 \\
\hline
\end{tabular}

(*) $m / z$ for quantification. 




Figure 3. Fragmentation mechanism of paraquat in the ionization chamber.

\subsection{Influence of mobile phase characteristics on chromatographic separation of diquat and paraquat}

\subsubsection{Effect of buffer concentration in solvent $A$}

The variabilities of retention time $\left(\mathrm{T}_{\mathrm{r}}\right)$, peak width $(\mathrm{W})$, and tailing factor $\left(\mathrm{F}_{\text {tailing }}\right)$ of diquat and paraquat affected by different buffer concentrations are exhibited in Table 3 The diquat and paraquat retention times increased from 0.4 minutes and 0.5 minutes, respectively, as the buffer concentrations increased from $5 \mathrm{mM}$ to $50 \mathrm{mM}$. This result is attributed to an increase in the buffer concentration that reduced the electrostatic interaction of the charged analyte on the zwitterion column. Diquat and paraquat retained on the column are affected by the simultaneous of two types of interactions, including i) the attractive interaction with the anion group outside the active layer and the repulsive interaction with the cation group inside; ii) increasing of the salt concentration can reduce both types of interactions, but the main effect was still to reduce electrostatic repulsion so that the analytes were better retained on the column [19]. Besides, Table 3 also exhibits that the chromatographic peak shape improved with the higher buffer concentration expressed through the decrease in peak width and $\mathrm{F}_{\text {tailing }}$ of analytes. We suggest that increasing the buffer concentrations can decrease secondary interactions, thereby improving the peak shape [20]. However, the buffer concentration is too high, the sensitivity of the signal will be affected by that expressed through the peak area. The peak areas of paraquat and diquat at different buffer concentrations are shown in Figure 4. The sensitivity of paraquat and diquat decreased when the buffer concentration increased from 5 to $50 \mathrm{mM}$. The cause can be due to the suppression in MS, the paraquat and diquat could be completed by the presence of overwhelming amounts of other ions before entering the quadrupole. In addition, Figure 4 also showed the standard deviation in peak area of repeated injections at a buffer concentration of $5 \mathrm{mM}$ was more extensive than other concentrations. We suggest that the buffer concentration of $5 \mathrm{mM}$ was too small, forming a weak buffer capacity. It was not enough to stabilize the mobile phase $\mathrm{pH}$, leading to the neutralization of the anion group on the unfavorable column that causing the analyte not to be entirely eluted from the chromatography column and would be further eluted in the next injection. That made the posterior injection area higher than the previous injection, leading to non-repeating peak areas between injections. Therefore, based on the obtained results, $10 \mathrm{mM}$ was chosen as the optimal buffer concentration due to the best stability, the satisfy the sensitivity requirement, and the acceptable peak shape. 
TABLE III. Retention time $\left(\mathrm{T}_{\mathrm{r}}\right)$, peak width $(\mathrm{W})$, tailing factor $\left(\mathrm{F}_{\text {tailing }}\right)$, and resolution $\left(\mathrm{R}_{\mathrm{s}}\right)$ of diquat and paraquat in the influence of different buffer concentrations

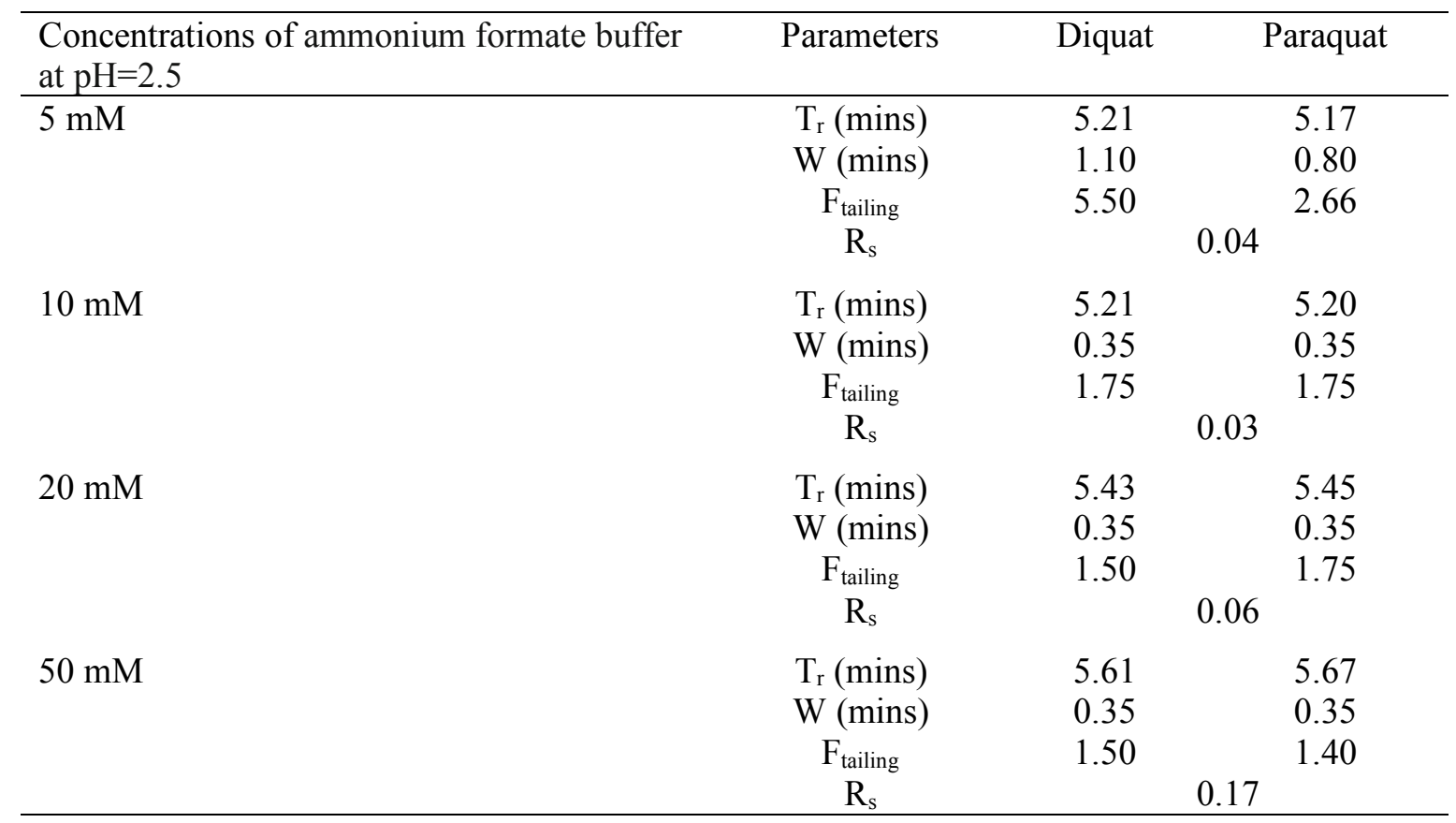

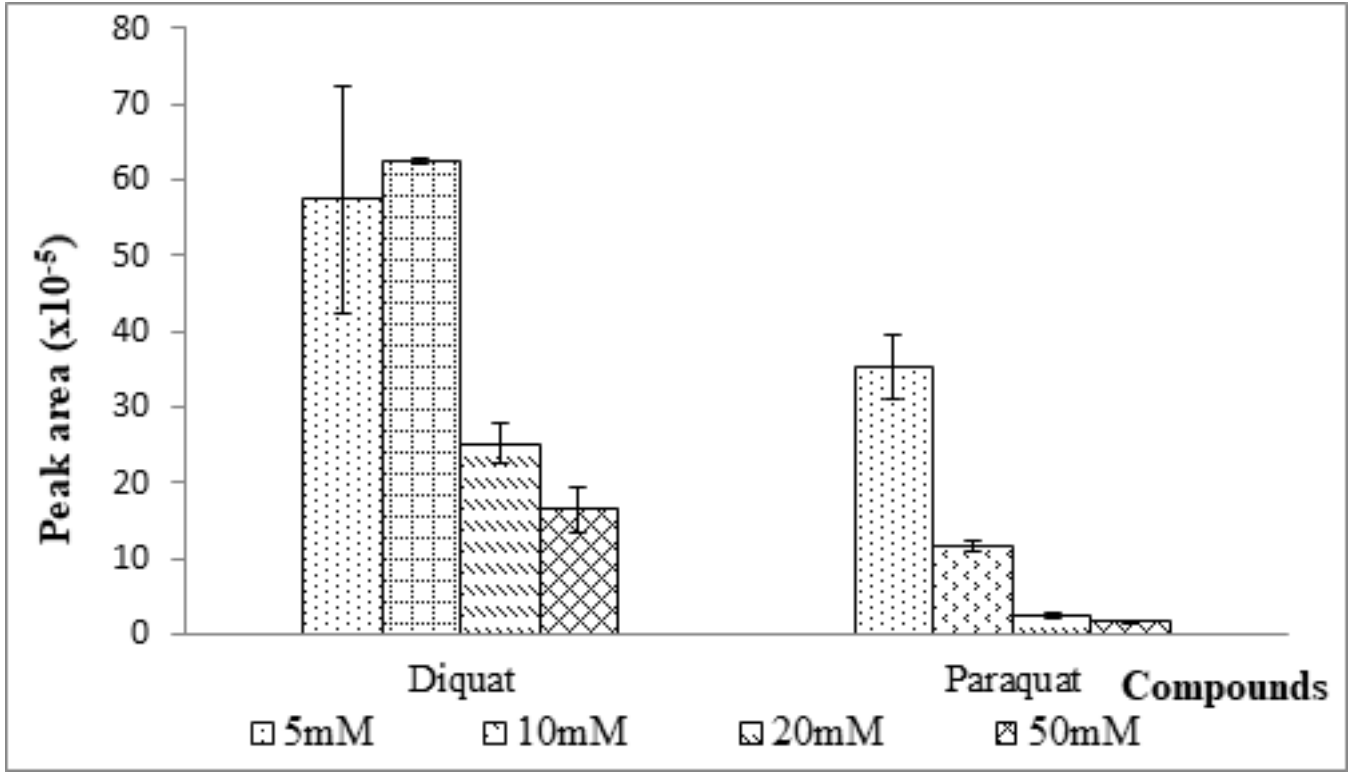

Figure 4. Peak area of diquat and paraquat at different buffer concentrations.

\subsubsection{Effect of buffer $\mathrm{pH}$}

The peak width (W) and $\mathrm{F}_{\text {tailing }}$ of diquat and paraquat at $\mathrm{pH}=3$ were more extensive than at $\mathrm{pH}=2.5$ (Table 4). The reason could be that incomplete neutralization in the column leads to some molecules of the paraquat and diquat remaining on the column, causing tailing and band broadening peaks. At a buffer $\mathrm{pH}$ of 2.5 , the anion group on the active layer exists almost entirely in a neutral form, thereby helping to elute these analytes better and improve the peak shape. Besides the presented influencing factors, buffer $\mathrm{pH}$ also affects MS and changes the sensitivity of the analytes. Consequently, the appearance of the chromatographic peak of diquat and paraquat at $\mathrm{pH}=5$ was not found. The reason may be that this $\mathrm{pH}$ range was not enough for the negatively charged group on the column's active layer to existing completely in neutral form. Figure 5 shows that the peak area of the paraquat was higher at $\mathrm{pH}=2.5$. The cause can be that adding a volatile acid such as formic acid to 
adjust the $\mathrm{pH}$ makes the solvent's volatility, resulting in better ionization efficiency. In the ESI source, the ionization efficiency may or may not be related to $\mathrm{pH}$ depending on the different physicochemical properties of the analytes [21]. That was the reason the sensitivity of paraquat was affected, and diquat was not when changing buffer $\mathrm{pH}$. From the obtained results, the appropriate buffer $\mathrm{pH}$ in solvent A was 2.5 because it gave the peak shape closest to the Gaussian peak and the higher sensitivity for paraquat.

TABLE IV. Retention time $\left(\mathrm{T}_{\mathrm{r}}\right)$, peak width $(\mathrm{W})$, tailing factor $\left(\mathrm{F}_{\text {tailing }}\right)$, and resolution $\left(\mathrm{R}_{\mathrm{s}}\right)$ of diquat and paraquat in different buffer $\mathrm{pHs}$

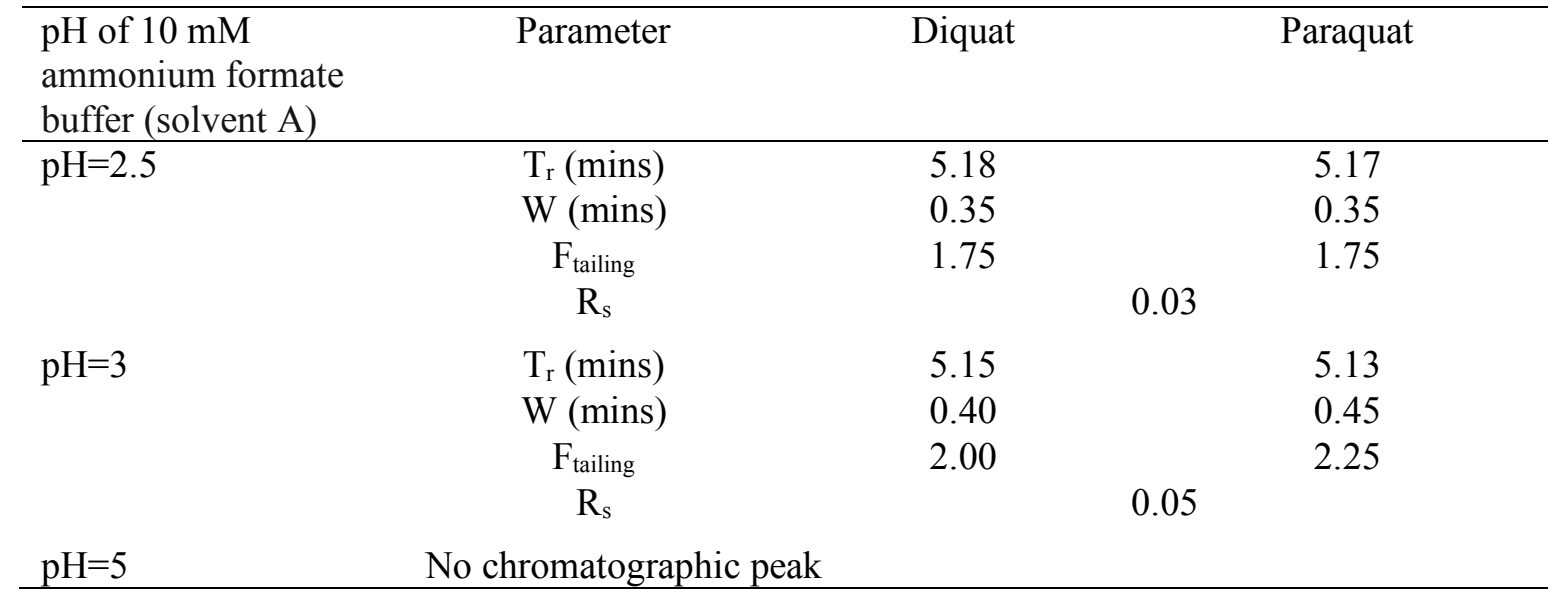

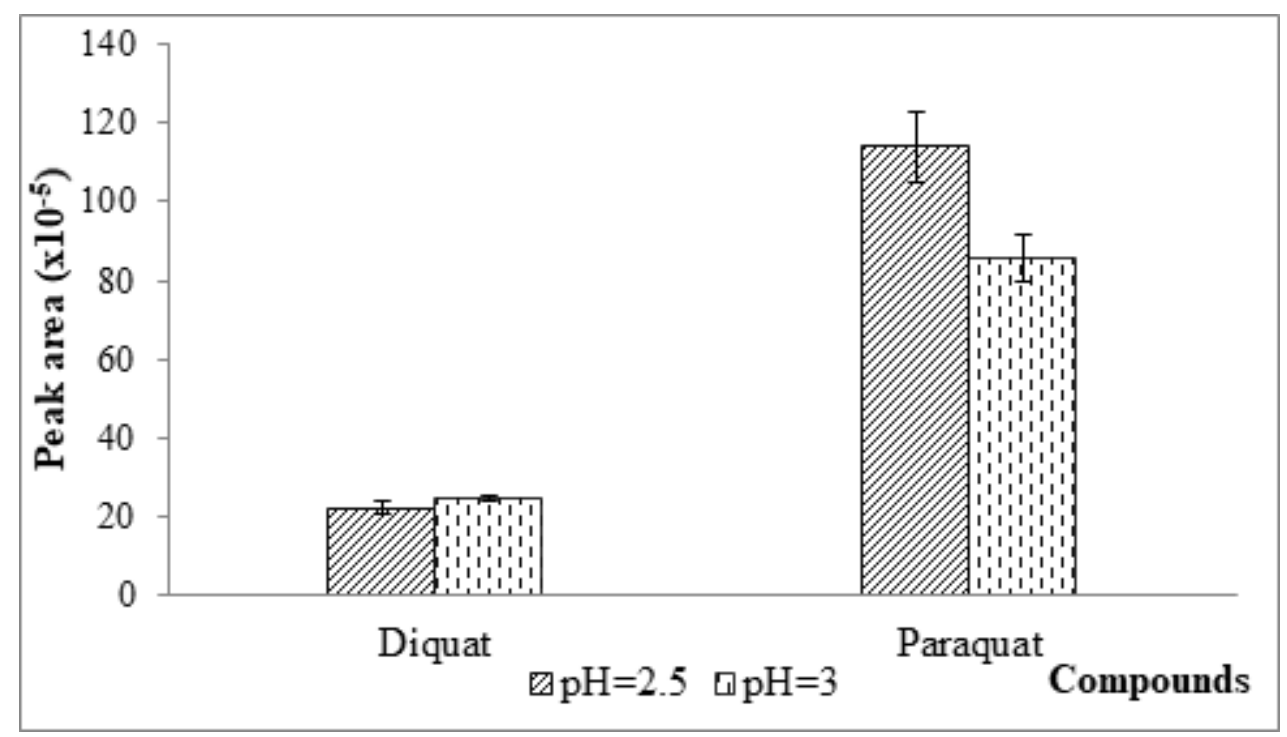

Figure 5. Peak area of diquat and paraquat at different buffer $\mathrm{pH}$.

\subsubsection{Effect of gradient elutions}

The specifics of the chromatographic peak of diquat and paraquat at different gradient elutions are presented in Table 5. The analytes were eluted from the column relatively quickly, simultaneously the tailing and band broadening peak paraquat. For the purpose of correcting the quick elution, gradient 2 was constructed by reducing the buffer percentage at an initial stage. The results obtained were more favorable in retention time, but the peak of the paraquat and diquat were still broader and more tailing due to being held for a long time on the column. Realizing that the peak shape of paraquat and diquat was insufficient because the highest percentage of the buffer solution used was not strong enough for elution, increasing the buffer percentage from $80 \%$ to $90 \%$ was applied. With a gradient 3 , the results were much improved, with the width and tail peak significantly reduced. In addition to the factors of retention time and peak shape for a gradient to be considered optimal, the equilibration time of the column after the analyte is eluted very importantly. For HILIC, columns 
need to have an equilibration time of about 20 times the column volume, specifically in this case $\sim 16$ minutes. The final compound comes out of the column at about $5 \mathrm{~min}$; hence the end time of gradient elution at 20 minutes was reasonable. As the above results, gradient 3 was selected as the optimal elution gradient.

TABLE V. Retention time $\left(\mathrm{T}_{\mathrm{r}}\right)$, peak width $(\mathrm{W})$, tailing factor $\left(\mathrm{F}_{\text {tailing }}\right)$, and resolution $\left(\mathrm{R}_{\mathrm{s}}\right)$ of diquat and paraquat in different gradient. elutions

\begin{tabular}{|c|c|c|c|}
\hline Gradient elutions & Parameters & Diquat & Paraquat \\
\hline \multirow[t]{4}{*}{ Gradient 1} & $\mathrm{~T}_{\mathrm{r}}(\mathrm{mins})$ & 3.93 & 3.89 \\
\hline & $\mathrm{W}$ (mins) & 0.35 & 0.45 \\
\hline & $\mathrm{F}_{\text {tailing }}$ & 1.75 & 2.25 \\
\hline & $\mathrm{R}_{\mathrm{s}}$ & \multicolumn{2}{|c|}{0.10} \\
\hline \multirow[t]{4}{*}{ Gradient 2} & $\mathrm{~T}_{\mathrm{r}}(\operatorname{mins})$ & 4.79 & 4.72 \\
\hline & $\mathrm{W}$ (mins) & 0.40 & 0.60 \\
\hline & $\mathrm{F}_{\text {tailing }}$ & 2.00 & 2.00 \\
\hline & $\mathrm{R}_{\mathrm{s}}$ & \multicolumn{2}{|c|}{0.14} \\
\hline \multirow[t]{4}{*}{ Gradient 3} & $\mathrm{~T}_{\mathrm{r}}(\mathrm{mins})$ & 5.21 & 5.18 \\
\hline & $\mathrm{W}$ (mins) & 0.35 & 0.35 \\
\hline & $\mathrm{F}_{\text {tailing }}$ & 1.75 & 1.75 \\
\hline & $\mathrm{R}_{\mathrm{s}}$ & \multicolumn{2}{|c|}{0.08} \\
\hline
\end{tabular}

\subsection{Method validation for analysis of diquat and paraquat}

The calibration curves of diquat and paraquat are shown in Figure 6. The linear range and correlation coefficient were $1-50 \mu \mathrm{g} / \mathrm{L}, 0.9997$ for diquat, and $1-200 \mu \mathrm{g} / \mathrm{L}, 0.9998$ for paraquat, respectively. The large linear ranges of diquat and paraquat allow the analysis of samples at various concentrations without sample dilution or enrichment. The results of the study showed that the matrix effect of diquat and paraquat alternate $-5.1 \%$ and $-4.2 \%$, which prove to have no significant matrix effects. Equation 1 was used to calculate the matrix effect.

$M E=\frac{a^{\prime}-a}{a} * 100$

Where,

ME: matrix effect (\%)

$a^{\prime}$ : the slope of calibration curve or peak area of analytes in rice matrix

a: the slope of calibration curve or peak area of analytes in a mixture of methanol: water $(6: 4=v / v)$


Figure 6. Standard curve in methanol: water mixture $(6: 4=v / v)$ containing $1 \%$ formic acid) of (a) diquat and (b) paraquat. 
The recoveries (Rec) of diquat and paraquat at the standard spiked concentration of $10 \mu \mathrm{g} / \mathrm{kg}$ were more than $70 \%$ for diquat and $88 \%$ for paraquat, respectively, with repeatabilities $\left(\mathrm{RSD}_{\mathrm{r}}\right.$ ), reproducibilities $\left(\mathrm{RSD}_{\mathrm{R}}\right)<15 \%$ (Table 6), satisfied the criteria of SANTE/11813/2017. It can be concluded that the MQL of diquat and paraquat was $10 \mu \mathrm{g} / \mathrm{kg}$, which is less than the maximum residue limits (MRL) specified by the EU for rice matrices. From MQL, the calculated MDL of those analytes was $3 \mu \mathrm{g} / \mathrm{kg}$.

TABLE VI. The recoveries $(\% \mathrm{R})$, repeatabilities $\left(\mathrm{RSD}_{\mathrm{r}}\right)$, reproducibilities $\left(\mathrm{RSD}_{\mathrm{R}}\right)$ of diquat and paraquat at the spiked concentration of $10 \mu \mathrm{g} / \mathrm{kg}$

\begin{tabular}{lcc}
\hline Parameters & Diquat $(10 \mu \mathrm{g} / \mathrm{kg})$ & Paraquat $(10 \mu \mathrm{g} / \mathrm{kg})$ \\
\hline$\% \mathrm{R}$ & 71.72 & 88.33 \\
$\mathrm{RSD}_{\mathrm{r}}(\%)$ & 6.41 & 11.22 \\
$\mathrm{RSD}_{\mathrm{R}}(\%)$ & 7.83 & 11.24 \\
\hline
\end{tabular}

The recoveries $(\mathrm{Rec})$, repeatability $\left(\mathrm{RSD}_{\mathrm{r}}\right)$, and reproducibility $\left(\mathrm{RSD}_{\mathrm{R}}\right)$ of standard spiked concentrations are shown in Table 7. These results satisfy SANTE/11813/2017 (\% H: 70-120\%; $\mathrm{RSD}_{\mathrm{r}}, \mathrm{RSD}_{\mathrm{R}}<20 \%$ ). We conclude that the method can be applied to analyze diquat and paraquat at various concentrations in rice.

TABLE VII. The recoveries (\% R), repeatabilities $\left(\mathrm{RSD}_{\mathrm{r}}\right)$, reproducibilities $\left(\mathrm{RSD}_{\mathrm{R}}\right)$ of diquat and paraquat at spiked concentrations of 20,50 , and $100 \mu \mathrm{g} / \mathrm{kg}$

\begin{tabular}{lccc}
\hline Spiked standard concentrations & Parameters & Diquat & Paraquat \\
\hline \multirow{2}{*}{$20 \mu \mathrm{g} / \mathrm{kg}$} & $\% \mathrm{R}$ & 71.84 & 79.01 \\
& $\mathrm{RSD}_{\mathrm{r}}(\%)$ & 7.72 & 1.71 \\
& $\mathrm{RSD}_{\mathrm{R}}(\%)$ & 8.60 & 6.84 \\
$50 \mu \mathrm{g} / \mathrm{kg}$ & $\% \mathrm{R}$ & 72.70 & 80.85 \\
& $\mathrm{RSD}_{\mathrm{r}}(\%)$ & 4.02 & 1.03 \\
& $\mathrm{RSD}_{\mathrm{R}}(\%)$ & 4.31 & 1.41 \\
& & & \\
$100 \mu \mathrm{g} / \mathrm{kg}$ & $\% \mathrm{R}$ & 70.31 & 81.53 \\
& $\mathrm{RSD}_{\mathrm{r}}(\%)$ & 1.04 & 4.03 \\
& $\mathrm{RSD}_{\mathrm{R}}(\%)$ & 1.65 & 3.20 \\
\hline
\end{tabular}

\subsection{Analysis of paraquat and diquat in rice from local markets}

The paraquat and diquat concentrations in selected rice samples were lower than MQL (i.e., $10 \mu \mathrm{g} / \mathrm{kg}$ ). The recovery and $\mathrm{RSD}_{\mathrm{r}}$ were described in Table 8 . Those values satisfied the SANTE/11813/2017. It proved that the results were not a false negative.

TABLE VIII. The recoveries (\% R) and RSD (\%) of diquat and paraquat in rice samples

\begin{tabular}{lcccc}
\hline \multirow{2}{*}{ Samples } & \multicolumn{2}{c}{ Diquat $(100 \mu \mathrm{g} / \mathrm{kg})$} & \multicolumn{2}{c}{ Paraquat $(100 \mu \mathrm{g} / \mathrm{kg})$} \\
\cline { 2 - 5 } & $\% \mathrm{R}$ & $\mathrm{RSD}_{\mathrm{r}}(\%)$ & $\mathrm{R}$ & $\mathrm{RSD}_{\mathrm{r}}(\%)$ \\
\hline Sample 1 & $70.83 \pm 3.96$ & 5.62 & $81.70 \pm 2.21$ & 2.74 \\
Sample 2 & $72.51 \pm 2.25$ & 3.13 & $83.94 \pm 6.63$ & 7.90 \\
Sample 3 & $71.11 \pm 4.83$ & 6.80 & $85.51 \pm 7.18$ & 8.44 \\
Sample 4 & $70.34 \pm 5.06$ & 7.21 & $82.65 \pm 4.96$ & 6.01 \\
Sample 5 & $71.60 \pm 3.51$ & 4.93 & $80.42 \pm 6.03$ & 7.54 \\
\hline
\end{tabular}




\section{CONCLUSION}

The conditions of chromatographic separation using HILIC Obelisc R column on UPLCMS/MS were optimized to analyze directly polar herbicides containing quaternary ammonium group typical diquat, paraquat in rice samples. The results showed that $10 \mathrm{mM}$ buffer concentration of ammonium formate at $\mathrm{pH}=2.5$ was the optimal condition for diquat and paraquat chromatographic separation on the UPLC-MS/MS. The method validation showed that the recoveries of diquat and paraquat were within the allowable range from 70 to $120 \%$, with $\mathrm{RSD}_{\mathrm{R}}$ and $\mathrm{RSD}_{\mathrm{R}}$ being less than $20 \%$ at the concentration ranges from 10 to $100 \mu \mathrm{g} / \mathrm{kg}$. The MQL of the investigated analytes was $10 \mu \mathrm{g} / \mathrm{kg}$, and this MQL was less than MRL according to EU regulations for rice. The developed method can be further applied to analyze diquat and paraquat in different rice samples. In addition, the combination with a quick, simple, and effective rice sample preparation based on the QuPPe method creates a premise to analyze other polar compounds in rice such as diminazene, isometamidium.

\section{References}

[1] M. Pateiro-Moure, M. Arias-Estévez, and J. Simal-Gándara, "Critical Review on the Environmental Fate of Quaternary Ammonium Herbicides in Soils Devoted to Vineyards", Environmental Science \& Technology, vol. 47 (10), pp. 4984-4998, 2013

[2] M. Pateiro-Moure, M. Arias-Estévez, E. López-Periago, E. Martínez-Carballo, and J. Simal-Gándara, "Occurrence and Downslope Mobilization of Quaternary Herbicide Residues in Vineyard-Devoted Soils", Bulletin of Environmental Contamination and Toxicology, vol. 80 (5), pp. 407-411, 2008

[3] D. I. Kolberg, D. Mack, M. Anastassiades, M. T. Hetmanski, R. J. Fussell, T. Meijer, and H. G. Mol, "Development and independent laboratory validation of a simple method for the determination of paraquat and diquat in potato, cereals and pulses", Anal Bioanal Chem, vol. 404 (8), pp. 2465-74, 2012.

[4] B. A. Bidlingmeyer, "Separation of ionic compounds by reversed-phase liquid chromatography an update of ion-pairing techniques", Journal of Chromatographic Science, vol. 18 (10), pp. 525-539, 1980.

[5] T. M. Chichila, and S. M. Walters, "Liquid chromatographic determination of paraquat and diquat in crops using a silica column with aqueous ionic mobile phase", Journal of the Association of Official Analytical Chemists, vol. 74 (6), pp. 961-967, 1991.

[6] M. A. Aramendía, V. Borau, F. Lafont, A. Marinas, J. M. Marinas, J. M. Moreno, J. M. Porras, and F. J. Urbano, "Determination of diquat and paraquat in olive oil by ion-pair liquid chromatographyelectrospray ionization mass spectrometry (MRM)", Food Chemistry, vol. 97 (1), pp. 181-188, 2006.

[7] K. Andrew, and B. Adam, "Rapid Separation of Paraquat and Diquat Using Hydrophilic Interaction Chromatography (HILIC) with LC/MS Detection", Agilent, 2017.

[8] S. Y. Michael, and M. J. Kevin, "Oasis ${ }^{\circledR}$ WCX: A Novel Mixed-Mode SPE Sorbent for LC-MS Determination of Paraquat and Other Quaternary Ammonium Compounds", Waters, pp. 44-45, 2004.

[9] T. Zou, P. He, J. Cao, and Z. Li, "Determination of paraquat in vegetables using HPLC-MS-MS", $J$ Chromatogr Sci, vol. 53 (2), pp. 204-9, 2015.

[10] P. G. Wang, and W. He, "Hydrophylic Interaction Liquid Chromatography and Advanced Applications". CPR Press Taylor \& Francis Group. pp. 89, 2019.

[11] M. X. Chen, Z. Y. Cao, Y. Jiang, and Z. W. Zhu, "Direct determination of glyphosate and its major metabolite, aminomethylphosphonic acid, in fruits and vegetables by mixed-mode hydrophilic interaction/weak anion-exchange liquid chromatography coupled with electrospray tandem mass spectrometry", J Chromatogr A, vol. 1272, pp. 90-9, 2013

[12] I. R. Pizzutti, I. G. M. Vela, A. de Kok, J. M. Scholten, J. V. Dias, C.D. Cardoso, G. Concenço, and R. Vivian, "Determination of paraquat and diquat: LC-MS method optimization and validation", Food chemistry, vol. 209, pp. 248-255, 2016

[13] European Commission. List of pesticide MRLs. http://ec.europa.eu/food/plant/pesticides/eu-pesticidesdatabase/public/?event=pesticide.residue.selection\&language=EN (Access online 17 January, 2017). 
[14] T. Axel and C. Ellanie, "Opportunities for Global Rice Research in a Changing World Technical bulletin Philippines". pp. 1, 2011.

[15] L. Pareja, A. R. Fernández-Alba, V. Cesio, and H. Heinzen, "Analytical methods for pesticide residues in rice", TrAC Trends in Analytical Chemistry, vol. 30 (2), pp. 270-291, 2011

[16] Technologies, SIELC. (Obelisc ${ }^{\mathrm{TM}}$ HPLC Columns - Liquid Separation Cell Technology, SIELC.

[17] M. Anastassiades, D. I. Kolberg, A. Benkenstein, E. Eichhorn, S. Zechmann, D. Mack, C. Wildgrube, I. Sigalov, D. Dörk, and A. Barth, "Quick method for the analysis of numerous highly polar pesticides in foods of plant origin via LC-MS/MS involving simultaneous extraction with methanol (QuPPe-method)", EU reference laboratory for pesticides requiring single residue methods (EURL-SRM). CVUA, Stuttgart, Germany, 2015.

[18] K. Wunnapuk, G. A. Medley, X. Liu, J. E. Grice, S. Jayasinghe, I. Gawarammana, N. A. Buckley, and M. S. Roberts, "Simple and sensitive liquid chromatography-tandem mass spectrometry methods for quantification of paraquat in plasma and urine: application to experimental and clinical toxicological studies", J Chromatogr B Analyt Technol Biomed Life Sci, vol. 879 (28), pp. 3047-52, 2011

[19] G. Greco, and T. Letzel, "Main interactions and influences of the chromatographic parameters in HILIC separations", J Chromatogr Sci, vol. 51 (7), pp. 684-93, 2013

[20] Y. Guo, and S. Gaiki, "Retention and selectivity of stationary phases for hydrophilic interaction chromatography", J Chromatogr A, vol. 1218 (35), pp. 5920-38, 2011

[21] J. Liigand, A. Laaniste, and A. Kruve, "pH Effects on Electrospray Ionization Efficiency", J Am Soc Mass Spectrom, vol. 28 (3), pp. 461-469, 2017

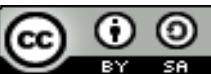

Jurnal IJCA is licensed under aÂ Creative Commons Attribution ShareAlike 4.0 